\title{
Chapter 26 \\ Path continuity connected with the notion of density
}

STANISŁAW KOWALCZYK, KATARZYNA NOWAKOWSKA

2010 Mathematics Subject Classification: 26A15, 54C30.

Key words and phrases: density of a set at a point, lower density, upper density, continuous functions, approximately continuous functions, path continuity.

\subsection{Preliminaries}

A. M. Bruckner, R. J. O'Malley and B. S.Thomson in [4] investigated the notion of a system of paths and studied a number of generalized derivatives. Properties of path continuous functions was intensively studied in [2], [6], [7], [8], [9], [10], [11], [13]. Similar approach to the notion of continuity was used in [5], [15].

We use this idea of path continuity for studying some notions of generalized continuity connected with density of a set at a point. Some basic properties of these classes of functions are presented.

First, we shall collect some of the notions and definitions which appear frequently in the sequel. The symbol $\lambda^{*}(E)$ denotes the Lebesgue outer measure of $E \subset \mathbb{R}$. In the whole paper we consider only real-valued functions defined on an open interval $I=(a, b)$.

Let $E$ be a measurable subset of $\mathbb{R}$ and let $x \in \mathbb{R}$. According to [3], the numbers

$$
\underline{d}^{+}(E, x)=\liminf _{t \rightarrow 0^{+}} \frac{\lambda(E \cap[x, x+t])}{t}
$$


and

$$
\bar{d}^{+}(E, x)=\limsup _{t \rightarrow 0^{+}} \frac{\lambda(E \cap[x, x+t])}{t}
$$

are called the right lower density of $E$ at $x$ and right upper density of $E$ at $x$. The left lower and upper densities of $E$ at $x$ are defined analogously. If

$$
\underline{d}^{+}(E, x)=\bar{d}^{+}(E, x) \quad \text { or } \quad \underline{d}^{-}(E, x)=\bar{d}^{-}(E, x),
$$

then we call these numbers the right density and left density of $E$ at $x$, respectively. The numbers

$$
\bar{d}(E, x)=\limsup _{\substack{t \rightarrow 0^{+} \\ k \rightarrow 0^{+}}} \frac{\lambda(E \cap[x-t, x+k])}{k+t}
$$

and

$$
\underline{d}(E, x)=\liminf _{\substack{t \rightarrow 0^{+} \\ k \rightarrow 0^{+}}} \frac{\lambda(E \cap[x-t, x+k])}{k+t}
$$

are called the upper and lower density of $E$ at $x$ respectively.

If $\bar{d}(E, x)=\underline{d}(E, x)$, we call this number the density of $E$ at $x$ and denote it by $d(E, x)$. It is clear that $E$ has the density at $x$ if and only if all four one-sided densities are equal.

When $d(E, x)=1$, we say that $x$ is a point of density of $E$.

Definition 26.1. [7] Let $E$ be a measurable subset of $\mathbb{R}$ and $x \in \mathbb{R}$.

1. For $0<\rho<1$ we say that $x$ is a point of $\rho$-type upper density of $E$ if $\bar{d}(E, x)>\rho$.

2. We say that $x$ is a point of 1-type upper density of $E$ if $\bar{d}(E, x)=1$.

Definition 26.2. [7] A real-valued function $f$ defined on an open interval $I$ is called $\rho$-upper continuous at $x$ provided that there is a measurable set $E \subset I$ such that the point $x$ is a point of $\rho$-type upper density of $E, x \in E$ and $f_{\mid E}$ is continuous at $x$. If $f$ is $\rho$-upper continuous at every point of $I$ we say that $f$ is $\rho$-upper continuous.

We will denote the class of all $\rho$-continuous functions defined on an open intervals $I$ by $\mathcal{U} \mathcal{C}_{\rho}$.

Definition 26.3. [13] Let $E$ be a measurable subset of $\mathbb{R}$ and $x \in \mathbb{R}$.

1. For $0<\Lambda \leq \rho<1$ we say that $x$ is a point of $[\Lambda, \rho]$-density of $E$ if $\bar{d}(E, x)>\rho$ and $\underline{d}(E, x)>\Lambda$. 
2. For $0<\Lambda<1$ we say that $x$ is a point of $[\Lambda, 1]$-density of $E$ if $\bar{d}(E, x)=1$ and $\underline{d}(E, x)>\Lambda$.

3. We say that $x$ is a point of $[1,1]$-density of $E$ if $\bar{d}(E, x)=\underline{d}(E, x)=1$.

Definition 26.4. [8], [13] Let $0<\lambda \leq \rho \leq 1$. A real-valued function $f$ defined on an open interval $I$ is called $[\Lambda, \rho]$-continuous at $x \in I$ provided that there is a measurable set $E \subset I$ such that $x$ is a point of $[\Lambda, \rho]$-density of $E, x \in E$ and $f_{\mid E}$ is continuous at $x$. If $f$ is $[\Lambda, \rho]$-continuous at each point of $I$ we say that $f$ is $[\Lambda, \rho]$-continuous.

We will denote the class of all $[\Lambda, \rho]$-continuous functions by $\mathcal{C}_{[\Lambda, \rho]}$. It is clear that $\mathcal{C}_{[1,1]}$ is exactly the class of approximately continuous functions.

Sometimes density of a set at a point is defined in other, symmetric, way. According to, for example [14], the lower density of $E$ at $x$ and upper density of $E$ at $x$ are defined as $\liminf _{t \rightarrow 0^{+}} \frac{\lambda(E \cap[x-t, x+t])}{2 t}$ and $\limsup _{t \rightarrow 0^{+}} \frac{\lambda(E \cap[x-t, x+t])}{2 t}$, respectively.

We will denote these densities by $s-\underline{d}^{+}(E, x)$ and $s-\bar{d}^{+}(E, x)$, respectively. If $s-\underline{d}(E, x)=s-\bar{d}(E, x)$ then we call this number the symmetric density of $E$ at $x$ and denote it by $s-d(E, x)$.

Corollary 26.5. For each measurable $E \subset \mathbb{R}$ and $x \in \mathbb{R}$ we have

1. $s-\bar{d}(E, x) \leq \bar{d}(E, x)$,

2. $s-\underline{d}(E, x) \geq \bar{d}(E, x)$,

3. $x$ is a point of the density of $E$, if and only if $x$ is a point of symmetric density of $E$.

Definition 26.6. Let $E$ be a measurable subset of $\mathbb{R}$ and $x \in \mathbb{R}$.

1. For $0<\rho<1$, then we say that $x$ is a point of $s \rho$-type upper density of $E$ if $s-\bar{d}(E, x)>\rho$.

2. We say that $x$ is a point of s-1-type upper density of $E$ if $s-\bar{d}(E, x)=1$.

Definition 26.7. A real-valued function $f$, defined on an open interval $I$, is called $s \rho$-upper continuous at $x$, provided that there is a measurable set $E \subset I$ such that the point $x$ is a point of $s \rho$-type upper density of $E, x \in E$ and $f_{\mid E}$ is continuous at $x$. If $f$ is $s \rho$-upper continuous at every point of $I$, we say that $f$ is $s \rho$-upper continuous.

We will denote the class of all $\rho$-continuous functions defined on an open intervals $I$ by $s \mathcal{U} \mathcal{C}_{\rho}$.

Definition 26.8. Let $E$ be a measurable subset of $\mathbb{R}$ and $x \in \mathbb{R}$. 
1. For $0<\Lambda \leq \rho<1$ we say that $x$ is a point of $s-[\Lambda, \rho]$-density of $E$ if $s-\bar{d}(E, x)>\rho$ and $s-\underline{d}(E, x)>\Lambda$.

2. For $0<\Lambda<1$ we say that $x$ is a point of $s$-[ $[\Lambda, 1]$-density of $E$ if $s-\bar{d}(E, x)=1$ and $s-\underline{d}(E, x)>\Lambda$.

3. We say that $x$ is a point of s-[1,1]-density of $E$ if $s-\bar{d}(E, x)=\mathrm{s}-\underline{d}(E, x)=1$.

Definition 26.9. Let $0<\Lambda \leq \rho \leq 1$. A real-valued function $f$ defined on an open interval $I$ is called s- $[\Lambda, \rho]$-continuous at $x \in I$, provided that there is a measurable set $E \subset I$ such that $x$ is a point of s-[$[\Lambda, \rho]$-density of $E, x \in E$ and $f_{\mid E}$ is continuous at $x$. If $f$ is s- $[\Lambda, \rho]$-continuous at each point of $I$, we say that $f$ is $\mathrm{s}-[\Lambda, \rho]$-continuous.

We will denote the class of all s- $[\Lambda, \rho]$-continuous functions by $s \mathcal{C}_{[\Lambda, \rho]}$.

Corollary 26.10. $\mathcal{C}_{a p} \subset \mathcal{C}_{[\Lambda, \rho]} \cap s \mathcal{C}_{[\Lambda, \rho]}$ for each $0<\Lambda \leq \rho \leq 1$ and $\mathcal{C}_{a p}=$ $\mathcal{C}_{[1,1]}=s \mathcal{C}_{[1,1]}$.

\subsection{Basic properties}

Lemma 26.11. Let $0<\Lambda \leq \rho \leq 1$. The following inclusions are obvious.

1. $\mathcal{C}_{[\Lambda, \rho]} \subset \mathcal{U} \mathcal{C}_{\rho}$,

2. $s \mathcal{C}_{[\Lambda, \rho]} \subset s \mathcal{U C}_{\rho}$,

3. $s \mathcal{C}_{[\Lambda, \rho]} \subset \mathcal{U C}_{\rho}$,

4. $\mathcal{U U C}_{\rho} \subset \mathcal{U C} \mathcal{C}_{\rho}$.

Theorem 26.12. Let $0<\rho \leq 1$. Then each function $f$ from $\mathcal{U C}_{\rho}$ is measurable.

Proof. Assume that there exists $f \in \mathcal{U C}_{\rho}$ which is not measurable. Then we can find a number $a \in \mathbb{R}$ for which at least one of the sets $\{x \in I: f(x)<a\}$, $\{x \in I: f(x)>a\}$ is nonmeasurable. Without loss of generality we may assume that the set $\{x \in I: f(x)<a\}$ is nonmeasurable. Denote

$$
A=\{x \in I: f(x)<a\}, \quad B=\{x \in I: f(x) \geq a\} .
$$

It is obvious that $B=I \backslash A$ is also nonmeasurable. Consider a measurable sets $A_{1} \subset A, B_{1} \subset B$ such that $A \backslash A_{1}$ and $B \backslash B_{1}$ do not contain a measurable set of positive measure. Therefore $A \backslash A_{1}$ and $B \backslash B_{1}$ are nonmeasurable sets. Let

$$
F=\left(A \backslash A_{1}\right) \cup\left(B \backslash B_{1}\right)=I \backslash\left(A_{1} \cup B_{1}\right) .
$$


Then $F$ is measurable. Let $\Phi_{d}(F)$ be a set of all density points of $F$. By the well-known Lebesgue Density Theorem, $\lambda\left(F \backslash \Phi_{d}(F)\right)=0$. Therefore there exists $x_{0} \in\left(A \backslash A_{1}\right) \cap \Phi_{d}(F)$.

Since $f$ is $\rho$-upper continuous at $x_{0}$, it follows that there exists a measurable set $E \subset \mathbb{R}$ such that $x_{0} \in E, \bar{d}\left(E, x_{0}\right)>\rho$ and $f_{\mid E}$ is continuous at $x_{0}$. Since $x_{0} \in A$, we have $f\left(x_{0}\right)<a$. Therefore it is possible to find $\delta>0$ such that $E \cap\left(x_{0}-\delta, x_{0}+\delta\right) \subset A$. Let $E^{\prime}=E \cap\left(x_{0}-\delta, x_{0}+\delta\right)$. Hence $x_{0} \in E^{\prime}, f_{\mid E^{\prime}}$ is continuous at $x_{0}, E^{\prime} \subset A$ and

$$
\bar{d}\left(E^{\prime}, x_{0}\right)=\bar{d}\left(E, x_{0}\right)>\rho>0 .
$$

We have

$$
E^{\prime}=\left(E^{\prime} \cap A_{1}\right) \cup\left(E^{\prime} \cap\left(A \backslash A_{1}\right)\right) .
$$

Since $E^{\prime}$ and $E^{\prime} \cap A_{1}$ are measurable, $E^{\prime} \cap\left(A \backslash A_{1}\right)$ is measurable, too. Hence $\lambda\left(E^{\prime} \cap\left(A \backslash A_{1}\right)\right)=0$. Moreover,

$$
\bar{d}\left(E^{\prime} \cap A_{1}, x_{0}\right)=1-\underline{d}\left(\mathbb{R} \backslash\left(E^{\prime} \cap A_{1}\right), x_{0}\right) \leq 1-\underline{d}\left(F, x_{0}\right)=1-1=0,
$$

because $\left(E^{\prime} \cap A_{1}\right) \cap F=\emptyset$. It follows that

$$
\bar{d}\left(E^{\prime}, x_{0}\right) \leq \bar{d}\left(E^{\prime} \cap A, x_{0}\right)+\bar{d}\left(E^{\prime} \cap\left(A \backslash A_{1}\right), x_{0}\right)=0+0=0,
$$

contradicting (26.1). Thus the assumption that $f$ may be nonmeasurable is false.

Corollary 26.13. All considered classes of functions $\mathcal{C}_{[\Lambda, \rho]}, s \mathcal{C}_{[\Lambda, \rho]}, \mathcal{U} \mathcal{C}_{\rho}$ and ${ }_{\mathcal{H C}} \rho$ consist of Lebesgue measurable functions.

Lemma 26.14. Let $0<\rho \leq 1, x \in \mathbb{R}$ and let $\left\{E_{n}: n \in \mathbb{N}\right\}$ be a decreasing family of measurable sets such that $\bar{d}\left(E_{n}, x\right) \geq \rho$ for $n \geq 1$. Then there exists a measurable set $E$ such that $\bar{d}(E, x) \geq \rho$ and for each $n \in \mathbb{N}$ there exists $\delta_{n}>0$ for which $E \cap\left[x-\delta_{n}, x+\delta_{n}\right] \subset E_{n}$.

Proof. By assumptions, $\bar{d}^{+}\left(E_{n}, x\right) \geq \rho$ or $\bar{d}^{-}\left(E_{n}, x\right) \geq \rho$ for each $n$. Hence there exists an infinite sequence $\left\{E_{n_{k}}: k \in \mathbb{N}\right\}$ such that $\bar{d}^{+}\left(E_{n_{k}}, x\right) \geq \rho$ for all $k \geq 1$ or $\bar{d}^{-}\left(E_{n_{k}}, x\right) \geq \rho$ for all $k \geq 1$. Without loss of generality we may assume that the first possibility occurs. Then $\bar{d}^{+}\left(E_{n}, x\right) \geq \rho$ for all $n \geq 1$, because $\left\{E_{n}: n \in \mathbb{N}\right\}$ is a decreasing family.

Let $x_{1}>x$ be any point for which $\frac{\lambda\left(E_{1} \cap\left[x, x_{1}\right]\right)}{x_{1}-x}>\rho\left(1-\frac{1}{2}\right)$ and $x_{1}-x<1$. Next, we can find $x<x_{2}<x_{1}$ such that

$$
\frac{\lambda\left(E_{1} \cap\left[x_{2}, x_{1}\right]\right)}{x_{1}-x}>\rho\left(1-\frac{1}{2}\right), \quad \frac{\lambda\left(E_{2} \cap\left[x, x_{2}\right]\right)}{x_{2}-x}>\rho\left(1-\frac{1}{4}\right) \text { and } x_{2}-x<\frac{1}{2} \text {. }
$$


Assume that points $x_{1}, x_{2}, \ldots, x_{n}$ are chosen, $x<x_{n}<\ldots<x_{1}, \frac{\lambda\left(E_{i-1} \cap\left[x_{i}, x_{i-1}\right]\right)}{x_{i-1}-x}>$ $\rho\left(1-\frac{1}{2^{i-1}}\right)$ for $i=2, \ldots, n, \frac{\lambda\left(E_{n} \cap\left[x, x_{n}\right]\right)}{x_{n}-x}>\rho\left(1-\frac{1}{2^{n}}\right)$ and $x_{n}-x<\frac{1}{n}$. Then there exists $x<x_{n+1}<x_{n}$ such that

$$
\frac{\lambda\left(E_{n} \cap\left[x_{n+1}, x_{n}\right]\right)}{x_{n}-x}>\rho\left(1-\frac{1}{2^{n}}\right), \frac{\lambda\left(E_{n+1} \cap\left[x, x_{n+1}\right]\right)}{x_{n+1}-x}>\rho\left(1-\frac{1}{2^{n+1}}\right)
$$

and $x_{n+1}-x<\frac{1}{n+1}$.

We have constructed inductively a decreasing sequence $\left\{x_{n}\right\}_{n \geq 1}$ such that

$$
\frac{\lambda\left(E_{n} \cap\left[x_{n+1}, x_{n}\right]\right)}{x_{n}-x}>\rho\left(1-\frac{1}{2^{n}}\right) \text { for } n \geq 1 \text {. }
$$

$$
\begin{aligned}
& \text { Let } E=\bigcup_{n=1}^{\infty}\left(E_{n} \cap\left[x_{n+1}, x_{n}\right]\right) \cup\{x\} \text {. Since } \\
& \limsup _{n \rightarrow \infty} \frac{\lambda\left(E \cap\left[x, x_{n}\right]\right)}{x_{n}-x} \geq \limsup _{n \rightarrow \infty} \frac{\lambda\left(E_{n} \cap\left[x_{n+1}, x_{n}\right]\right)}{x_{n}-x} \geq \lim _{n \rightarrow \infty}\left(\rho-\frac{1}{2^{n}}\right)=\rho,
\end{aligned}
$$

we have $\bar{d}(E, x) \geq \rho$.

By definition of $E$, for each $n$ there exists $\delta_{n}=x_{n}-x>0$ such that

$$
E \cap\left[x-\delta_{n}, x+\delta_{n}\right]=E \cap\left[x, x_{n}\right] \subset E_{n} .
$$

The proof is complete.

We will give a condition equivalent to $\rho$-upper continuity at a point $x$.

Theorem 26.15. Let $0<\rho \leq 1$ and let $f: I \rightarrow \mathbb{R}$ be a measurable function. Then $f$ is $\rho$-upper continuous at $x \in I$ if and only if

$$
\lim _{\varepsilon \rightarrow 0^{+}} \bar{d}(\{y \in I:|f(x)-f(y)|<\varepsilon\}, x)>\rho \quad \text { if } \quad 0<\rho<1
$$

or

$$
\bar{d}(\{y \in I:|f(x)-f(y)|<\varepsilon\}, x)=1 \quad \text { for all } \varepsilon>0 \quad \text { if } \quad \rho=1 .
$$

Proof. Assume that $f$ is $\rho$-upper continuous at $x$. Let $E \subset I$ be a measurable set such that $x \in E, f_{\mid E}$ is continuous at $x$ and $\bar{d}(E, x)>\rho$ for $\rho<1$, or $\bar{d}(E, x)=1$ if $\rho=1$. Since $f_{\mid E}$ is continuous at $x$, for each $\varepsilon>0$ we can find $\delta>0$ such that $[x-\delta, x+\delta] \cap E \subset\{y:|f(x)-f(y)|<\varepsilon\}$. Hence

$\bar{d}(\{y \in I:|f(x)-f(y)|<\varepsilon\}, x) \geq \bar{d}(\{y \in E:|f(x)-f(y)|<\varepsilon\}, x)=\bar{d}(E, x)$ 
for each $\varepsilon>0$. Therefore

$$
\lim _{\varepsilon \rightarrow 0^{+}} \bar{d}(\{y \in I:|f(x)-f(y)|<\varepsilon\}, x) \geq \bar{d}(E, x)>\rho \quad \text { if } \rho<1
$$

or

$\bar{d}(\{y \in I:|f(x)-f(y)|<\varepsilon\}, x) \geq \bar{d}(E, x)=1 \quad$ for each $\varepsilon>0, \quad$ if $\rho=1$.

Finally, assume that

$$
\rho_{1}=\lim _{\varepsilon \rightarrow 0^{+}} \bar{d}(\{y \in I:|f(x)-f(y)|<\varepsilon\}, x)>\rho \quad \text { if } \rho<1
$$

or

$$
\bar{d}(\{y \in I:|f(x)-f(y)|<\varepsilon\}, x)=1 \text { for each } \varepsilon>0 \quad \text { if } \rho=1 .
$$

Applying Lemma 26.14 for sets $E_{n}=\left\{y \in I:|f(x)-f(y)|<\frac{1}{n}\right\}$, we can construct a measurable set $E$ such that $x \in E$,

$$
\bar{d}(E, x) \geq \rho_{1}>\rho \quad \text { if } \quad 0<\rho<1 \quad \text { or } \quad \bar{d}(E, x)=1 \quad \text { if } \quad \rho=1
$$

and for each $n$ there exists $\delta_{n}>0$ for which $E \cap\left[x-\delta_{n}, x+\delta_{n}\right] \subset E_{n}$. The last condition implies that $f_{\mid E}$ is continuous at $x$. It follows that $f$ is $\rho$-upper continuous at $x$.

Corollary 26.16.

$$
\bigcap_{0<\rho<1} \mathcal{U C}_{\rho}=\mathcal{U C}_{1}
$$

Lemma 26.17. Let $0<\rho \leq 1, x \in \mathbb{R}$ and let $\left\{E_{n}: n \in \mathbb{N}\right\}$ be a decreasing family of measurable sets such that $s-\bar{d}\left(E_{n}, x\right) \geq \rho$ for $n \geq 1$. Then there exists a measurable set $E$ such that $s-\bar{d}(E, x) \geq \rho$ and for each $n \in \mathbb{N}$ there exists $\delta_{n}>0$ for which $E \cap\left[x-\delta_{n}, x+\delta_{n}\right] \subset E_{n}$.

Proof. Let $\delta_{1}>0$ be such that $\frac{\lambda\left(E_{1} \cap\left[x-\delta_{1}, x+\delta_{1}\right]\right)}{2 \delta_{1}}>\rho\left(1-\frac{1}{2}\right)$ and $\delta_{1}<1$. Next, we can find $\delta_{2} \in\left(0, \delta_{1}\right)$ such that

$$
\begin{gathered}
\frac{\lambda\left(E_{1} \cap\left(\left[x-\delta_{1}, x-\delta_{2}\right] \cup\left[x+\delta_{2}, x+\delta_{1}\right]\right)\right)}{2 \delta_{1}}>\rho\left(1-\frac{1}{2}\right), \\
\frac{\lambda\left(E_{2} \cap\left[x-\delta_{2}, x+\delta_{2}\right]\right)}{2 \delta_{2}}>\rho\left(1-\frac{1}{4}\right)
\end{gathered}
$$

and $\delta_{2}<\frac{1}{2}$. Assume that real positive numbers $\delta_{1}, \delta_{2}, \ldots, \delta_{n}$ are chosen, $\delta_{n}<\delta_{n-1}<\ldots<\delta_{1}$, 


$$
\frac{\lambda\left(E_{i-1} \cap\left(\left[x-\delta_{i-1}, x-\delta_{i}\right] \cup\left[x+\delta_{i}, x+\delta_{i-1}\right]\right)\right.}{2 \delta_{i-1}-x}>\rho\left(1-\frac{1}{2^{i-1}}\right)
$$

for $i=2, \ldots, n, \frac{\lambda\left(E_{n} \cap\left[x-\delta_{n}, x+\delta_{n}\right]\right)}{2 \delta_{n}}>\rho\left(1-\frac{1}{2^{n}}\right)$ and $\delta_{n}<\frac{1}{n}$. Then there exists $\delta_{n+1} \in\left(0, \delta_{n}\right)$ such that $\frac{\lambda\left(E_{n} \cap\left(\left[x-\delta_{n}, x-\delta_{n+1}\right] \cup\left[x+\delta_{n+1}, x+\delta_{n}\right]\right)\right.}{2 \delta_{n}}>\rho\left(1-\frac{1}{2^{n}}\right)$, $\frac{\lambda\left(E_{n+1} \cap\left[x-\delta_{n+1}, x+\delta_{n+1}\right]\right)}{2 \delta_{n+1}}>\rho\left(1-\frac{1}{2^{n+1}}\right)$ and $\delta_{n+1}<\frac{1}{n+1}$.

We have constructed inductively a decreasing sequence $\left\{\delta_{n}\right\}_{n \geq 1}$ of positive numbers such that for $n \geq 1$

$$
\frac{\lambda\left(E_{n} \cap\left(\left[x-\delta_{n}, x-\delta_{n+1}\right] \cup\left[x+\delta_{n+1}, x+\delta_{n}\right]\right)\right)}{2 \delta_{n}}>\rho\left(1-\frac{1}{2^{n}}\right) .
$$

Let $E=\{x\} \cup \bigcup_{n=1}^{\infty}\left(E_{n} \cap\left(\left[x-\delta_{n}, x-\delta_{n+1}\right] \cup\left[x+\delta_{n+1}, x+\delta_{n}\right]\right)\right)$. Since

$$
\begin{aligned}
& \limsup _{n \rightarrow \infty} \frac{\lambda\left(E \cap\left[x-\delta_{n}, x+\delta_{n}\right]\right)}{2 \delta_{n}} \geq \\
\geq & \limsup _{n \rightarrow \infty} \frac{\lambda\left(E_{n} \cap\left(\left[x-\delta_{n}, x-\delta_{n+1}\right] \cup\left[x+\delta_{n+1}, x+\delta_{n}\right]\right)\right)}{2 \delta_{n}} \geq \lim _{n \rightarrow \infty}\left(\rho-\frac{1}{2^{n}}\right)=\rho,
\end{aligned}
$$

we have $s-\bar{d}(E, x) \geq \rho$.

By definition of $E$, we have $E \cap\left[x-\delta_{n}, x+\delta_{n}\right]=E \cap\left[x, x_{n}\right] \subset E_{n}$ for each $n$. The proof is complete.

Now, we can give a condition equivalent to $s \rho$-upper continuity at a point $x$.

Theorem 26.18. Let $0<\rho \leq 1$ and let $f: I \rightarrow \mathbb{R}$ be a measurable function. Then $f$ is $s \rho$-upper continuous at $x \in I$ if and only if

$$
\lim _{\varepsilon \rightarrow 0^{+}} s-\bar{d}(\{y \in I:|f(x)-f(y)|<\varepsilon\}, x)>\rho \quad \text { if } \quad 0<\rho<1
$$

or

$$
s-\bar{d}(\{y \in I:|f(x)-f(y)|<\varepsilon\}, x)=1 \quad \text { for all } \varepsilon>0 \quad \text { if } \quad \rho=1 .
$$

Proof. The proof is analogous to the proof of Theorem 26.15. The unique difference is that we use Lemma 26.17 instead of Lemma 26.14.

Corollary 26.19.

$$
\bigcap_{0<\rho<1} \mathcal{S U C}_{\rho}=s \mathcal{U} \mathcal{C}_{1}
$$

Remark 26.20. Conditions stated in Theorem 26.15 and 26.15 are similar to the general condition of path continuity discussed in [15, Theorem 14.3]. 
Lemma 26.21. Let $\Lambda \in(0,1]$. If $E$ is a measurable subset of $\mathbb{R}$ and $\underline{d}^{+}(E, x) \geq$ $\Lambda$ then for each $n \in \mathbb{N}$ there exists $\varepsilon_{n}>0$ such that

$$
\frac{\lambda\left(E \cap\left[x+\frac{a}{2 n}, x+b\right]\right)}{b}>\Lambda-\frac{1}{n}
$$

for each $0<a<b<\varepsilon_{n}$.

Proof. Fix any $n \in \mathbb{N}$. Since $\underline{d}^{+}(E, x) \geq \Lambda$, there exists $\varepsilon_{n} \in(0,1)$ such that $\frac{\lambda(E \cap[x, x+c])}{c}>\Lambda-\frac{1}{2 n}$ for each $c \in\left(0, \varepsilon_{n}\right)$. If $0<a<b<\varepsilon_{n}$, then

$$
\begin{aligned}
\lambda\left(E \cap\left[x+\frac{a}{2 n}, x+b\right]\right)=\lambda(E \cap[x, x+b])-\lambda & \left(E \cap\left[x, x+\frac{a}{2 n}\right]\right) \geq \\
& \geq b\left(1-\frac{1}{2 n}\right)-\frac{a}{2 n}>b \Lambda-\frac{b}{n} .
\end{aligned}
$$

Hence $\frac{\lambda\left(E \cap\left[x+\frac{a}{2 n}, x+b\right]\right)}{b}>\Lambda-\frac{1}{n}$.

Lemma 26.22. Let $0<\rho \leq 1$ and let $x \in \mathbb{R}$. Assume that $E \subset \mathbb{R}$ is measurable and $\bar{d}^{+}(E, x) \geq \rho$. For every $n \in \mathbb{N}$ there exists decreasing sequence $\left\{\alpha_{m}\right\}_{m \in \mathbb{N}}$ of positive reals converging to 0 such that

$$
\frac{\lambda\left(E \cap\left[x+\frac{\alpha_{m}}{2 n}, x+\alpha_{m}\right]\right)}{\alpha_{m}}>\rho-\frac{1}{n}
$$

for each $m \in \mathbb{N}$.

Proof. Fix $n \in \mathbb{N}$. Since $\bar{d}^{+}(E, x) \geq \rho$, there exists a decreasing sequence $\left\{\beta_{m}\right\}_{m \in \mathbb{N}}$ of positive reals such that

$$
\lim _{m \rightarrow \infty} \frac{\lambda\left(E \cap\left[x, x+\beta_{m}\right]\right)}{\beta_{m}}=\bar{d}^{+}(E, x) \geq \rho .
$$

Then we can find $m_{0}$ such that $\frac{\lambda\left(E \cap\left[x, x+\beta_{m}\right]\right)}{\beta_{m}}>\rho-\frac{1}{n}$ for all $m \geq m_{0}$. Hence,

$$
\frac{\lambda\left(E \cap\left[x+\frac{\beta_{m}}{2 m}, x+\beta_{m}\right]\right)}{\beta_{m}} \geq \frac{\lambda\left(E \cap\left[x, x+\beta_{m}\right]\right)}{\beta_{m}}-\frac{\lambda\left(E \cap\left[x, x+\frac{\beta_{m}}{2 m}\right]\right)}{\beta_{m}}>\rho-\frac{1}{n}
$$

for each $m \geq m_{0}$. Then the sequence $\left\{\alpha_{m}\right\}_{m \in \mathbb{N}}$, where $\alpha_{m}=\beta_{m+m_{0}}$ for $m \in \mathbb{N}$, has all the required properties.

Lemma 26.23. Let $0<\Lambda \leq \rho \leq 1$ and let $\left\{E_{n}\right\}_{n \in \mathbb{N}}$ be a decreasing sequence of measurable sets such that $x \in \bigcap_{n=1}^{\infty} E_{n}, \underline{d}^{+}\left(E_{n}, x\right) \geq \Lambda$ and $\bar{d}\left(E_{n}, x\right) \geq \rho$ for all $n \in \mathbb{N}$. Then there exists a measurable set $E$ such that $\underline{d}(E, x) \geq \Lambda, \bar{d}(E, x) \geq \rho$, $x \in E$ and for each $n \in \mathbb{N}$ there exists $\delta_{n}>0$ for which $E \cap\left[x-\delta_{n}, x+\delta_{n}\right] \subset E_{n}$ 
Proof. As in the proof of Lemma 26.14, we can assume that $\bar{d}^{+}\left(E_{n}, x\right) \geq \rho$ for all $n$. By Lemma 26.21, for each $n \in \mathbb{N}$ there exists $\varepsilon_{n}>$ such that

$$
\frac{\lambda\left(E_{n} \cap\left[x+\frac{a}{2 n}, x+b\right]\right)}{b}>\Lambda-\frac{1}{n}
$$

for all $0<a, b<\varepsilon_{n}$. By Lemma 26.22, for each $n \in \mathbb{N}$ we can find decreasing sequence $\left\{\alpha_{m}^{n}\right\}_{m \in \mathbb{N}}$ such that

$$
\frac{\lambda\left(E_{n} \cap\left[x+\frac{\alpha_{m}^{n}}{2 n}, x+\alpha_{m}^{n}\right]\right)}{\alpha_{m}^{n}}>\rho-\frac{1}{n}
$$

for all $m, n \in \mathbb{N}$. We will construct inductively a sequence of positive reals $\left\{a_{n}\right\}_{n \in \mathbb{N}}$ such that for each $n \in \mathbb{N}$

1. $a_{n}<\varepsilon_{n}$,

2. $a_{n+1}<\frac{a_{n}}{2 n}$,

3. there exists $m_{n} \in \mathbb{N}$ for which $\left[\frac{\alpha_{m_{n}}^{n}}{2 n}, \alpha_{m_{n}}^{n}\right] \subset\left[a_{n+1}, a_{n}\right]$.

Choose any $a_{1}<\varepsilon_{1}$. Assume that we have chosen $a_{1}, \ldots, a_{n}$ satisfying conditions 1) - 3). Then we can find $m_{n} \in \mathbb{N}$ such that $\alpha_{m_{n}}^{n}<a_{n}$. Now, we can take an arbitrary $a_{n+1} \in\left(0, \min \left\{\varepsilon_{n+1}, \frac{\alpha_{m_{n}}^{n}}{2 n}\right\}\right)$. Put

$$
F=\bigcup_{n=1}^{\infty}\left(E_{n} \cap\left[x+a_{n+2}, x+a_{n+1}\right]\right) .
$$

Let $y \in\left[x, x+a_{2}\right], y=x+c$. Then $c \in\left[a_{n+1}, a_{n}\right]$ for some $n$. Since $\frac{a_{n+1}}{2 n}>$ $a_{n+2}$, we have

$$
\begin{aligned}
& F \cap\left[a_{n+2}, x+c\right] \supset\left(E_{n} \cap\left[x+a_{n+2}, x+a_{n+1}\right]\right) \cup \\
& \cup\left(E_{n-1} \cap\left[x+a_{n+1}, x+c\right]\right) \supset E_{n} \cap\left[x+\frac{a_{n+1}}{2 n}, x+c\right] .
\end{aligned}
$$

Hence

$$
\lambda(F \cap[x, x+c]) \geq \lambda\left(E_{n} \cap\left[x+\frac{a_{n+1}}{2 n}, x+c\right]\right)>c\left(\Lambda-\frac{1}{n}\right)
$$

and $\frac{\lambda(F \cap[x, x+c])}{c}>\Lambda-\frac{1}{n}$. Therefore $\underline{d}^{+}(F, x) \geq \Lambda$.

On the other hand,

$$
F \cap\left[x, x+\alpha_{m_{n}}^{n}\right] \supset E_{n} \cap\left[x+a_{n+2}, x+\alpha_{m_{n}}^{n}\right] \supset E_{n} \cap\left[x+\frac{a_{n+1}}{2 n}, x+\alpha_{m_{n}}^{n}\right] .
$$

Therefore $\frac{\lambda\left(F \cap\left[x, x+\alpha_{m_{n}}^{n}\right]\right)}{\alpha_{m_{n}}^{n}}>\rho-\frac{1}{n}$ for $n \in \mathbb{N}$ and 


$$
\bar{d}^{+}(F, x) \geq \limsup _{n \rightarrow \infty} \frac{\lambda\left(F \cap\left[x, x+\alpha_{m_{n}}^{n}\right]\right)}{\alpha_{m_{n}}^{n}} \geq \limsup _{n \rightarrow \infty}\left(\rho-\frac{1}{n}\right)=\rho .
$$

Finally, we can easily see that for each $n \in \mathbb{N}$ we can choose $\delta_{n}=a_{n+1}$ for which $E \cap\left[x, x+\delta_{n}\right] \subset E_{n}$.

Similarly, we can construct a measurable set $G \subset(-\infty, x)$ such that $\underline{d}^{-}(G, x) \geq \lambda$ and for each $n \in \mathbb{N}, G \cap\left[x-\delta_{n}, x\right] \subset E_{n}$ for some positive $\delta_{n}$. Then the set $E=F \cup G \cup\{x\}$ has all the required properties.

Theorem 26.24. Let $0<\Lambda \leq \rho<1$. A measurable function $f: I \rightarrow \mathbb{R}$ is $[\Lambda, \rho]$ continuous at $x_{0}$ if and only if

$$
\lim _{\varepsilon \rightarrow 0^{+}} \underline{d}\left(\left\{x \in I:\left|f(x)-f\left(x_{0}\right)\right|<\varepsilon\right\}, x_{0}\right)>\Lambda
$$

and

$$
\lim _{\varepsilon \rightarrow 0^{+}} \bar{d}\left(\left\{x \in I:\left|f(x)-f\left(x_{0}\right)\right|<\varepsilon\right\}, x_{0}\right)>\rho .
$$

Proof. asasAssume that a measurable $f$ is $[\Lambda, \rho]$-continuous at $x_{0}$. Then there exists measurable $E \subset \mathbb{R}$ such that $x_{0} \in E, \underline{d}\left(E, x_{0}\right)>\Lambda, \bar{d}\left(E, x_{0}\right)>\rho$ and $f_{\mid E}$ is continuous at $x_{0}$. By the continuity of $f$ at $x_{0}$, for each $\varepsilon>0$ we can find $\delta>0$ for which $\left[x_{0}-\delta, x_{0}+\delta\right] \subset\left\{x:\left|f(x)-f\left(x_{0}\right)\right|<\varepsilon\right\}$. Hence

$\underline{d}\left(\left\{x:\left|f(x)-f\left(x_{0}\right)\right|<\varepsilon\right\}, x_{0}\right) \geq \underline{d}\left(\left\{x \in E:\left|f(x)-f\left(x_{0}\right)\right|<\varepsilon\right\}, x_{0}\right)=\underline{d}\left(E, x_{0}\right)$

and

$\bar{d}\left(\left\{x:\left|f(x)-f\left(x_{0}\right)\right|<\varepsilon\right\}, x_{0}\right) \geq \bar{d}\left(\left\{x \in E:\left|f(x)-f\left(x_{0}\right)\right|<\varepsilon\right\}, x_{0}\right)=\bar{d}\left(E, x_{0}\right)$.

Therefore

$$
\lim _{\varepsilon \rightarrow 0^{+}} \underline{d}\left(\left\{x \in I:\left|f(x)-f\left(x_{0}\right)\right|<\varepsilon\right\}, x_{0}\right)>\Lambda
$$

and

$$
\lim _{\varepsilon \rightarrow 0^{+}} \bar{d}\left(\left\{x \in I:\left|f(x)-f\left(x_{0}\right)\right|<\varepsilon\right\}, x_{0}\right)>\rho .
$$

Now assume that

$$
\Lambda_{1}=\lim _{\varepsilon \rightarrow 0^{+}} \underline{d}\left(\left\{x \in I:\left|f(x)-f\left(x_{0}\right)\right|<\varepsilon\right\}, x_{0}\right)>\Lambda
$$

and

$$
\rho_{1}=\lim _{\varepsilon \rightarrow 0^{+}} \bar{d}\left(\left\{x \in I:\left|f(x)-f\left(x_{0}\right)\right|<\varepsilon\right\}, x_{0}\right)>\rho .
$$

Applying Lemma 26.23 for $E_{n}=\left\{x \in I:\left|f(x)-f\left(x_{0}\right)\right|<\frac{1}{n}\right\}$, we can find a measurable set $E \subset \mathbb{R}$ such that $x_{0} \in E, \underline{d}\left(E, x_{0}\right) \geq \Lambda_{1}>\Lambda, \bar{d}\left(E, x_{0}\right) \geq \rho_{1}>\rho$ 
and for each $n \in \mathbb{N}$ there exists $\delta_{n}>0$ such that $E \cap\left[x_{0}-\delta_{n}, x_{0}+\delta_{n}\right] \subset E_{n}$. Hence, $f_{\mid E}$ is continuous at $x_{0}$ and $f$ is $[\Lambda, \rho]$-continuous.

Theorem 26.25. Let $0<\Lambda<1$. A measurable function $f: I \rightarrow \mathbb{R}$ is $[\Lambda, 1]$ continuous at $x_{0}$ if and only if

$$
\lim _{\varepsilon \rightarrow 0^{+}} \underline{d}\left(\left\{x \in I:\left|f(x)-f\left(x_{0}\right)\right|<\varepsilon\right\}, x_{0}\right)>\Lambda
$$

and

$$
\bar{d}\left(\left\{x \in I:\left|f(x)-f\left(x_{0}\right)\right|<\varepsilon\right\}, x_{0}\right)=1
$$

for each $\varepsilon>0$.

Proof. Assume that a measurable $f$ is $[\Lambda, 1]$-continuous at $x_{0}$. Then there exists a measurable $E \subset \mathbb{R}$ such that $x_{0} \in E, \underline{d}\left(E, x_{0}\right)>\Lambda, \bar{d}\left(E, x_{0}\right)=1$ and $f_{\mid E}$ is continuous at $x_{0}$. By the continuity of $f$ at $x_{0}$, for each $\varepsilon>0$ we can find $\delta>0$ for which $E \cap\left[x_{0}-\delta, x_{0}+\delta\right] \subset\left\{x:\left|f(x)-f\left(x_{0}\right)\right|<\varepsilon\right\}$. Hence

$\underline{d}\left(\left\{x:\left|f(x)-f\left(x_{0}\right)\right|<\varepsilon\right\}, x_{0}\right) \geq \underline{d}\left(\left\{x \in E:\left|f(x)-f\left(x_{0}\right)\right|<\varepsilon\right\}, x_{0}\right)=\underline{d}\left(E, x_{0}\right)$

and

$\bar{d}\left(\left\{x:\left|f(x)-f\left(x_{0}\right)\right|<\varepsilon\right\}, x_{0}\right) \geq \bar{d}\left(\left\{x \in E:\left|f(x)-f\left(x_{0}\right)\right|<\varepsilon\right\}, x_{0}\right)=\bar{d}\left(E, x_{0}\right)$.

Therefore

$$
\lim _{\varepsilon \rightarrow 0^{+}} \underline{d}\left(\left\{x \in I:\left|f(x)-f\left(x_{0}\right)\right|<\varepsilon\right\}, x_{0}\right)>\Lambda
$$

and

$$
\bar{d}\left(\left\{x \in I:\left|f(x)-f\left(x_{0}\right)\right|<\varepsilon\right\}, x_{0}\right)=1 .
$$

Now assume that

$$
\Lambda_{1}=\lim _{\varepsilon \rightarrow 0^{+}} \underline{d}\left(\left\{x \in I:\left|f(x)-f\left(x_{0}\right)\right|<\varepsilon\right\}, x_{0}\right)>\Lambda
$$

and

$$
\lim _{\varepsilon \rightarrow 0^{+}} \bar{d}\left(\left\{x \in I:\left|f(x)-f\left(x_{0}\right)\right|<\varepsilon\right\}, x_{0}\right)=1
$$

for each $\varepsilon>0$. Applying Lemma 26.23 for $E_{n}=\left\{x \in I:\left|f(x)-f\left(x_{0}\right)\right|<\frac{1}{n}\right\}$, we can find a measurable set $E \subset \mathbb{R}$ such that $x_{0} \in E, \underline{d}\left(E, x_{0}\right) \geq \lambda_{1}>\lambda$, $\bar{d}\left(E, x_{0}\right)=1$ and for each $n \in \mathbb{N}$ there exists $\delta_{n}>0$ such that $E \cap\left[x_{0}-\delta_{n}, x_{0}+\right.$ $\left.\delta_{n}\right] \subset E_{n}$. Hence $f_{\mid E}$ is continuous at $x_{0}$ and $f$ is $[\Lambda, \rho]$-continuous.

\section{Corollary 26.26.}

$$
\bigcap_{0<\Lambda \leq \rho<1} \mathcal{C}_{[\Lambda, \rho]}=\mathcal{C}_{a p}
$$


Lemma 26.27. Let $\Lambda \in(0,1]$. If $E \subset \mathbb{R}$ is measurable and $s-\underline{d}^{+}(E, x) \geq \Lambda$ then for each $n \in \mathbb{N}$ there exists $\varepsilon_{n}>0$ such that

$$
\frac{\lambda\left(E \cap\left(\left[x-b, x-\frac{a}{2 n}\right] \cup\left[x+\frac{a}{2 n}, x+b\right]\right)\right)}{2 b}>\Lambda-\frac{1}{n}
$$

for each $0<a<b<\varepsilon_{n}$.

Proof. Fix any $n \in \mathbb{N}$. There exists $\varepsilon_{n} \in(0,1)$ such that $\frac{\lambda(E \cap[x-c, x+c])}{2 c}>\Lambda-\frac{1}{2 n}$ for each $c \in\left(0, \varepsilon_{n}\right)$. If $0<a<b<\varepsilon_{n}$, then

$$
\begin{aligned}
\lambda\left(E \cap\left(\left(\left[x-b, x-\frac{a}{2 n}\right] \cup\left[x+\frac{a}{2 n}, x+b\right]\right)\right)=\lambda(E \cap[x-b, x+b])-\right. \\
-\lambda\left(E \cap\left[x-\frac{a}{2 n}, x+\frac{a}{2 n}\right]\right) \geq 2 b\left(1-\frac{1}{2 n}\right)-\frac{2 a}{2 n}>b \Lambda-\frac{b}{n} .
\end{aligned}
$$

Lemma 26.28. Let $0<\rho \leq 1$ and let $x \in \mathbb{R}$. Assume that $E \subset \mathbb{R}$ is measurable and $s-\bar{d}^{+}(E, x) \geq \rho$. For every $n \in \mathbb{N}$ there exists a decreasing sequence $\left\{\alpha_{m}\right\}_{m \in \mathbb{N}}$ of positive reals converging to 0 such that

$$
\frac{\lambda\left(E \cap\left(\left[x-\alpha_{m}, x-\frac{\alpha_{m}}{2 n}\right] \cup\left[x+\frac{\alpha_{m}}{2 n}, x+\alpha_{m}\right]\right)\right)}{2 \alpha_{m}}>\rho-\frac{1}{n}
$$

for each $m \in \mathbb{N}$.

Proof. Fix $n \in \mathbb{N}$. There exists a decreasing sequence $\left\{\beta_{m}\right\}_{m \in \mathbb{N}}$ of positive reals such that

$$
\lim _{m \rightarrow \infty} \frac{\lambda\left(E \cap\left[x-\beta_{m}, x+\beta_{m}\right]\right)}{2 \beta_{m}}=s-\bar{d}^{+}(E, x) \geq \rho .
$$

Then we can find $m_{0}$ such that $\frac{\lambda\left(E \cap\left[x-\beta_{m}, x+\beta_{m}\right]\right)}{2 \beta_{m}}>\rho-\frac{1}{n}$ for all $m \geq m_{0}$. Hence

$$
\begin{aligned}
\frac{\lambda\left(E \cap\left(\left[x-\beta_{m}, x-\frac{\beta_{m}}{2 m}\right] \cup\left[x+\frac{\beta_{m}}{2 m}, x+\beta_{m}\right]\right)\right)}{2 \beta_{m}} \geq \\
\quad \geq \frac{\lambda\left(E \cap\left[x-\beta_{m}, x+\beta_{m}\right]\right)}{2 \beta_{m}}-\frac{\lambda\left(E \cap\left[x-\frac{\beta_{m}}{2 m}, x+\frac{\beta_{m}}{2 m}\right]\right)}{2 \beta_{m}}>\rho-\frac{1}{n}
\end{aligned}
$$

for each $m \geq m_{0}$. Then the sequence $\left\{\alpha_{m}\right\}_{m \in \mathbb{N}}$, where $\alpha_{m}=\beta_{m+m_{0}}$ for $m \in \mathbb{N}$, has all the required properties.

Lemma 26.29. Let $0<\Lambda \leq \rho \leq 1$ and let $\left\{E_{n}\right\}_{n \in \mathbb{N}}$ be a decreasing sequence of measurable sets such that $x \in \bigcap_{n=1}^{\infty} E_{n}, s-\underline{d}^{+}\left(E_{n}, x\right) \geq \Lambda$ and $s-\bar{d}\left(E_{n}, x\right) \geq \rho$ 
for all $n \in \mathbb{N}$. Then there exists a measurable set $E$ such that $s-\underline{d}(E, x) \geq \Lambda$, $s-\bar{d}(E, x) \geq \rho, x \in E$ and for each $n \in \mathbb{N}$ there exists a positive real $\delta_{n}>0$ for which $E \cap\left[x-\delta_{n}, x+\delta_{n}\right] \subset E_{n}$

Proof. By Lemma 26.27, for each $n \in \mathbb{N}$ there exists $\varepsilon_{n}>$ such that

$$
\frac{\lambda\left(E_{n} \cap\left(\left[x-b, x-\frac{a}{2 n}\right] \cup\left[x+\frac{a}{2 n}, x+b\right]\right)\right)}{2 b}>\Lambda-\frac{1}{n}
$$

for all $0<a<b<\varepsilon_{n}$. By Lemma 26.28, for each $n \in \mathbb{N}$ we can find decreasing sequence $\left\{\alpha_{m}^{n}\right\}_{m \in \mathbb{N}}$ such that

$$
\frac{\lambda\left(E_{n} \cap\left(\left[x-\alpha_{m}^{n}, x-\frac{\alpha_{m}^{n}}{2 n}\right] \cup\left[x+\frac{\alpha_{m}^{n}}{2 n}, x+\alpha_{m}^{n}\right]\right)\right)}{2 \alpha_{m}^{n}}>\rho-\frac{1}{n}
$$

for all $m, n \in \mathbb{N}$. As in the proof of Lemma 26.23, we will construct inductively a sequence of positive reals $\left\{a_{n}\right\}_{n \in \mathbb{N}}$ such that for each $n \in \mathbb{N}$

1. $a_{n}<\varepsilon_{n}$,

2. $a_{n+1}<\frac{a_{n}}{2 n}$,

3. there exists $m_{n} \in \mathbb{N}$ for which $\left[\frac{\alpha_{m_{n}}^{n}}{2 n}, \alpha_{m_{n}}^{n}\right] \subset\left[a_{n+1}, a_{n}\right]$.

Let $c \in\left[a_{n+1}, a_{n}\right]$ for some $n$. Since $\frac{a_{n+1}}{2 n}>a_{n+2}$, we have

$$
\begin{aligned}
& F \cap\left(\left[x-c, x-a_{n+2}\right] \cup\left[x+a_{n+2}, x+c\right]\right) \supset \\
& \supset\left(E_{n} \cap\left(\left[x-a_{n+1}, x-a_{n+2}\right] \cup\left[x+a_{n+2}, x+a_{n+1}\right]\right)\right) \cup \\
& \cup\left(E_{n-1} \cap\left(\left[x-c, x-a_{n+1}\right] \cup\left[x+a_{n+1}, x+c\right]\right)\right) \supset \\
& \supset E_{n} \cap\left(\left[x-c, x-\frac{a_{n+1}}{2 n}\right] \cup\left[x+\frac{a_{n+1}}{2 n}, x+c\right]\right) .
\end{aligned}
$$

Hence

$$
\lambda(F \cap[x-c, x+c]) \geq \lambda\left(E_{n} \cap\left(\left[x-c, x-\frac{a_{n+1}}{2 n}\right] \cup\left[x+\frac{a_{n+1}}{2 n}, x+c\right]\right)\right)>c\left(\Lambda-\frac{1}{n}\right)
$$

and $\frac{\lambda(F \cap[x-c, x+c])}{c}>\Lambda-\frac{1}{n}$. Therefore $s-\underline{d}^{+}(F, x) \geq \Lambda$.

On the other hand,

$$
\begin{aligned}
F \cap\left[x-\alpha_{m_{n}}^{n}, x+\alpha_{m_{n}}^{n}\right] \supset E_{n} \cap\left(\left[x-\alpha_{m_{n}}^{n}, x-a_{n+2}\right] \cup\left[x+a_{n+2}, x+\alpha_{m_{n}}^{n}\right]\right) \supset \\
\supset E_{n} \cap\left(\left[x-\frac{\alpha_{n+1}}{2 n}, x-\alpha_{m_{n}}^{n}\right] \cup\left[x+\alpha_{m_{n}}^{n}, x+\frac{\alpha_{n+1}}{2 n}\right]\right) .
\end{aligned}
$$

It follows that $\frac{\lambda\left(F \cap\left[x-\alpha_{m_{n}}^{n}, x+\alpha_{m_{n}}^{n}\right]\right)}{2 \alpha_{m_{n}}^{n}}>\rho-\frac{1}{n}$ for $n \in \mathbb{N}$ and 


$$
s-\bar{d}^{+}(F, x) \geq \limsup _{n \rightarrow \infty} \frac{\lambda\left(F \cap\left[x-\alpha_{m_{n}}^{n}, x+\alpha_{m_{n}}^{n}\right]\right)}{2 \alpha_{m_{n}}^{n}} \geq \limsup _{n \rightarrow \infty}\left(\rho-\frac{1}{n}\right)=\rho .
$$

Finally, we can easily see that for each $n \in \mathbb{N}$ we can choose $\delta_{n}=a_{n+1}$ for which $E \cap\left[x-\delta_{n}, x+\delta_{n}\right] \subset E_{n}$.

Theorem 26.30. Let $0<\Lambda \leq \rho<1$. A measurable function $f: I \rightarrow \mathbb{R}$ is $s[\Lambda, \rho]$-continuous at $x_{0}$, if and only if

$$
\lim _{\varepsilon \rightarrow 0^{+}} s-\underline{d}\left(\left\{x \in I:\left|f(x)-f\left(x_{0}\right)\right|<\varepsilon\right\}, x_{0}\right)>\Lambda
$$

and

$$
\lim _{\varepsilon \rightarrow 0^{+}} s-\bar{d}\left(\left\{x \in I:\left|f(x)-f\left(x_{0}\right)\right|<\varepsilon\right\}, x_{0}\right)>\rho .
$$

Proof. Assume that a measurable $f$ is $s[\Lambda, \rho]$-continuous at $x_{0}$. Then there exists a measurable $E \subset I$ such that $x_{0} \in E, s-\underline{d}\left(E, x_{0}\right)>\Lambda, s-\bar{d}\left(E, x_{0}\right)>\rho$ and $f_{\mid E}$ is continuous at $x_{0}$. By the continuity of $f$ at $x_{0}$, for each $\varepsilon>0$ we can find $\delta>0$ for which $E \cap\left[x_{0}-\delta, x_{0}+\delta\right] \subset\left\{x:\left|f(x)-f\left(x_{0}\right)\right|<\varepsilon\right\}$. Hence

$$
\begin{aligned}
s-\underline{d}\left(\left\{x:\left|f(x)-f\left(x_{0}\right)\right|<\varepsilon\right\}, x_{0}\right) & \geq s-\underline{d}\left(\left\{x \in E:\left|f(x)-f\left(x_{0}\right)\right|<\varepsilon\right\}, x_{0}\right)= \\
& =s-\underline{d}\left(E, x_{0}\right)
\end{aligned}
$$

and

$$
\begin{aligned}
s-\bar{d}\left(\left\{x:\left|f(x)-f\left(x_{0}\right)\right|<\varepsilon\right\}, x_{0}\right) & \geq s-\bar{d}\left(\left\{x \in E:\left|f(x)-f\left(x_{0}\right)\right|<\varepsilon\right\}, x_{0}\right)= \\
& =s-\bar{d}\left(E, x_{0}\right) .
\end{aligned}
$$

Therefore

$$
\lim _{\varepsilon \rightarrow 0^{+}} s-\underline{d}\left(\left\{x \in I:\left|f(x)-f\left(x_{0}\right)\right|<\varepsilon\right\}, x_{0}\right)>\Lambda
$$

and

$$
\lim _{\varepsilon \rightarrow 0^{+}} s-\bar{d}\left(\left\{x \in I:\left|f(x)-f\left(x_{0}\right)\right|<\varepsilon\right\}, x_{0}\right)>\rho .
$$

Now assume that

$$
\Lambda_{1}=\lim _{\varepsilon \rightarrow 0^{+}} s \underline{d}\left(\left\{x \in I:\left|f(x)-f\left(x_{0}\right)\right|<\varepsilon\right\}, x_{0}\right)>\Lambda
$$

and

$$
\rho_{1}=\lim _{\varepsilon \rightarrow 0^{+}} s-\bar{d}\left(\left\{x \in I:\left|f(x)-f\left(x_{0}\right)\right|<\varepsilon\right\}, x_{0}\right)>\rho .
$$

Applying Lemma 26.29 for $E_{n}=\left\{x \in I:\left|f(x)-f\left(x_{0}\right)\right|<\underline{\frac{1}{n}}\right\}$, we can find a measurable set $E \subset I$ such that $x_{0} \in E, s-\underline{d}\left(E, x_{0}\right) \geq \lambda_{1}>\lambda, s-\bar{d}\left(E, x_{0}\right) \geq \rho_{1}>\rho$ 
and for each $n \in \mathbb{N}$ there exists $\delta_{n}>0$ such that $E \cap\left[x_{0}-\delta_{n}, x_{0}+\delta_{n}\right] \subset E_{n}$. Hence $f_{\mid E}$ is continuous at $x_{0}$. Thus $f$ is $s[\Lambda, \rho]$-continuous at $x_{0}$.

Theorem 26.31. Let $0<\Lambda<1$. A measurable function $f: I \rightarrow \mathbb{R}$ is $s$-[ $\Lambda, 1]$ continuous at $x_{0}$, if and only if

$$
\lim _{\varepsilon \rightarrow 0^{+}} s-\underline{d}\left(\left\{x \in I:\left|f(x)-f\left(x_{0}\right)\right|<\varepsilon\right\}, x_{0}\right)>\Lambda
$$

and

$$
s-\bar{d}\left(\left\{x \in I:\left|f(x)-f\left(x_{0}\right)\right|<\varepsilon\right\}, x_{0}\right)=1
$$

for each $\varepsilon>0$.

Proof. Assume that a measurable $f$ is $s$-[ $[\Lambda, 1]$-continuous at $x_{0}$. Then there exists measurable $E \subset \mathbb{R}$ such that $x_{0} \in E, s-\underline{d}\left(E, x_{0}\right)>\Lambda, s-\bar{d}\left(E, x_{0}\right)=1$ and $f_{\mid E}$ is continuous at $x_{0}$. By the continuity of $f$ at $x_{0}$, for each $\varepsilon>0$ we can find $\delta>0$ for which $E \cap\left[x_{0}-\delta, x_{0}+\delta\right] \subset\left\{x:\left|f(x)-f\left(x_{0}\right)\right|<\varepsilon\right\}$. Hence

$$
\begin{aligned}
s-\underline{d}\left(\left\{x:\left|f(x)-f\left(x_{0}\right)\right|<\varepsilon\right\}, x_{0}\right) & \geq s-\underline{d}\left(\left\{x \in E:\left|f(x)-f\left(x_{0}\right)\right|<\varepsilon\right\}, x_{0}\right)= \\
& =s-\underline{d}\left(E, x_{0}\right)
\end{aligned}
$$

and

$$
\begin{aligned}
s-\bar{d}\left(\left\{x:\left|f(x)-f\left(x_{0}\right)\right|<\varepsilon\right\}, x_{0}\right) & \geq s-\bar{d}\left(\left\{x \in E:\left|f(x)-f\left(x_{0}\right)\right|<\varepsilon\right\}, x_{0}\right)= \\
& =s-\bar{d}\left(E, x_{0}\right) .
\end{aligned}
$$

Therefore

$$
\lim _{\varepsilon \rightarrow 0^{+}} s-\underline{d}\left(\left\{x \in I:\left|f(x)-f\left(x_{0}\right)\right|<\varepsilon\right\}, x_{0}\right)>\Lambda
$$

and

$$
s-\bar{d}\left(\left\{x \in I:\left|f(x)-f\left(x_{0}\right)\right|<\varepsilon\right\}, x_{0}\right)=1 .
$$

Now assume that

$$
\Lambda_{1}=\lim _{\varepsilon \rightarrow 0^{+}} s-\underline{d}\left(\left\{x \in I:\left|f(x)-f\left(x_{0}\right)\right|<\varepsilon\right\}, x_{0}\right)>\Lambda
$$

and

$$
\lim _{\varepsilon \rightarrow 0^{+}} s-\bar{d}\left(\left\{x \in I:\left|f(x)-f\left(x_{0}\right)\right|<\varepsilon\right\}, x_{0}\right)=1
$$

for each $\varepsilon>0$. Applying Lemma 26.29 for $E_{n}=\left\{x \in I:\left|f(x)-f\left(x_{0}\right)\right|<\frac{1}{n}\right\}$, we can find a measurable set $E \subset \mathbb{R}$ such that $x_{0} \in E, \underline{d}\left(E, x_{0}\right) \geq \Lambda_{1}>\Lambda$, $\bar{d}\left(E, x_{0}\right)=1$ and for each $n \in \mathbb{N}$ there exists $\delta_{n}>0$ such that $E \cap\left[x_{0}-\delta_{n}, x_{0}+\right.$ $\left.\delta_{n}\right] \subset E_{n}$. Hence, $f_{\mid E}$ is continuous at $x_{0}$ and $f$ is $s[\Lambda, \rho]$-continuous. 


\section{Corollary 26.32.}

$$
\bigcap_{0<\Lambda \leq \rho<1} s \mathcal{C}_{[\Lambda, \rho]}=\mathcal{C}_{a p}
$$

Definition 26.33. We say that a real-valued function $f$ defined on an open interval $I$ has Denjoy property at $x_{0} \in I$, if it is measurable and

$$
\forall_{\varepsilon>0} \forall_{\delta>0} \quad \lambda\left(\left\{x \in\left(x_{0}-\delta, x_{0}+\delta\right):\left|f(x)-f\left(x_{0}\right)\right|<\varepsilon\right\}\right)>0 .
$$

We say that $f$ has Denjoy property, if it has Denjoy property at each point $x \in I$.

We will denote the class of all functions with Denjoy property by Den. By Theorem 26.12 and definition of $\mathcal{U} \mathcal{C}_{\rho}$ we have.

Corollary 26.34. Let $0<\rho<1$. If $f \in \mathcal{U C} \rho$ then $f$ has Denjoy property.

Corollary 26.35. By Lemma 26.11, all defined classes of functions have Denjoy property.

The diagram shows the relations among the considered classes of functions.

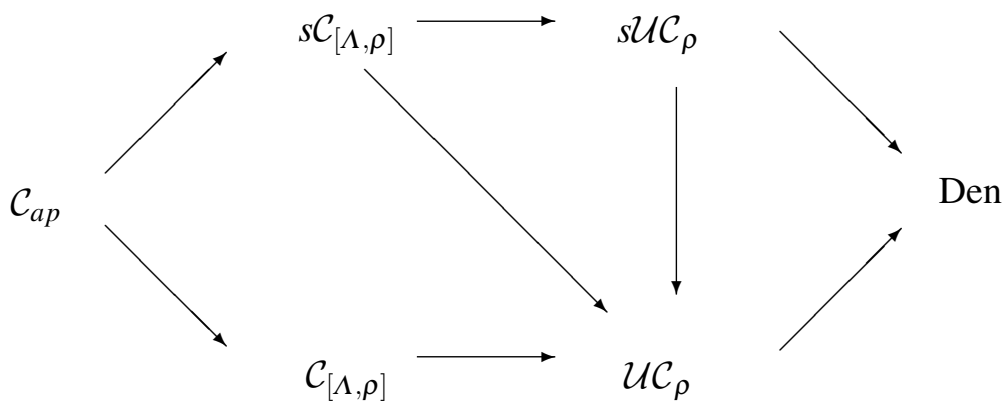

No other implication can be stated as a few examples show.

Example 26.36. There exists $f: \mathbb{R} \rightarrow \mathbb{R}$ such that $f \in\left(\mathcal{U C C}_{\rho} \cap \mathcal{U} \mathcal{C}_{\rho}\right) \backslash\left(s \mathcal{C}_{[\Lambda, \rho]} \cup\right.$ $\left.\mathcal{C}_{[\Lambda, \rho]}\right)$ for all $0<\rho \leq 1, \Lambda \in(0, \rho]$.

Let $\left\{\left[a_{n}, b_{n}\right]\right\}_{n \in \mathbb{N}}$ and $\left\{\left[c_{n}, d_{n}\right]\right\}_{n \in \mathbb{N}}$ be two sequences of closed intervals such that $0<\ldots d_{n+1}<c_{n}<a_{n}<b_{n}<d_{n}<\ldots<d_{1}, \bar{d}^{+}\left(\bigcup_{n=1}^{\infty}\left[a_{n}, b_{n}\right], 0\right)=1$, $\underline{d}^{+}\left(\bigcup_{n=1}^{\infty}\left[a_{n}, b_{n}\right], 0\right)=0$ and $\bar{d}^{+}\left(\bigcup_{n=1}^{\infty}\left(\left[c_{n}, a_{n}\right] \cup\left[b_{n}, d_{n}\right]\right), 0\right)=0$. Define $f: \mathbb{R} \rightarrow$ $\mathbb{R}$ letting

$$
f(x)=\left\{\begin{array}{l}
0 \text { for } x \in \bigcup_{n=1}^{\infty}\left(\left[a_{n}, b_{n}\right] \cup\left[-b_{n},-a_{n}\right]\right) \cup\{0\}, \\
1 \text { for } x \in \mathbb{R} \backslash\left(\{0\} \cup \bigcup_{n=1}^{\infty}\left(\left[c_{n}, d_{n}\right] \cup\left[-d_{n},-c_{n}\right]\right)\right), \\
\text { linear in intervals }\left[c_{n}, a_{n}\right],\left[b_{n}, d_{n}\right],\left[-a_{n},-c_{n}\right],\left[-d_{n},-b_{n}\right], n=1, \ldots
\end{array}\right.
$$


It is clear that $f$ is continuous at each point except at 0 . Let $E=\bigcup_{n=1}^{\infty}\left(\left[a_{n}, b_{n}\right] \cup\right.$ $\left.\left[-b_{n},-a_{n}\right]\right) \cup\{0\}$. Then $f_{\mid E}$ is constant and $\bar{d}(E, 0)=s-\bar{d}(E, 0)=1$. Therefore $f \in \mathcal{U U C}_{\rho} \cap \mathcal{U} \mathcal{C}_{\rho}$ for $\rho \in(0,1]$. On the other hand,

$$
\underline{d}(\{x:|f(x)|<1\}, 0)=s-\underline{d}(\{x:|f(x)|<1\}, 0)=1-\bar{d}(E, 0)=0 .
$$

Thus $f \notin s \mathcal{C}_{[\Lambda, \rho]} \cup \mathcal{C}_{[\Lambda, \rho]}$ for any $0<\rho \leq 1, \Lambda \in(0, \rho]$.

Example 26.37. For each $0<\Lambda<\rho \leq 1$ there exists $f: \mathbb{R} \rightarrow \mathbb{R}$ such that $f \in\left(\mathcal{U C}_{\rho} \cap \mathcal{C}_{[\Lambda, \rho]}\right) \backslash\left(s \mathcal{C}_{[\Lambda, \rho]} \cup \mathcal{U U C}_{\rho}\right)$.

Let $0<\Lambda<\rho \leq 1$. There exists $\alpha, \beta \in(0,1]$ such that $\alpha>\Lambda, \frac{\alpha+\beta}{2}<\rho$ and $\beta>\rho$ if $\rho<1$ or $\beta=1$ if $\rho=1$. Let $\left\{\left[a_{n}, b_{n}\right]\right\}_{n \in \mathbb{N}},\left\{\left[c_{n}, d_{n}\right]\right\}_{n \in \mathbb{N}},\left\{\left[a_{n}^{\prime}, b_{n}^{\prime}\right]\right\}_{n \in \mathbb{N}}$, $\left\{\left[c_{n}^{\prime}, d_{n}^{\prime}\right]\right\}_{n \in \mathbb{N}}$ be four sequences of closed intervals such that

$$
\begin{gathered}
\ldots<c_{n}^{\prime}<a_{n}^{\prime}<b_{n}^{\prime}<d_{n}^{\prime}<c_{n+1}^{\prime}<\ldots<0<\ldots d_{n+1}<c_{n}<a_{n}<b_{n}<d_{n}<\ldots, \\
d^{+}\left(\bigcup_{n=1}^{\infty}\left[a_{n}, b_{n}\right], 0\right)=\beta, d^{-}\left(\bigcup_{n=1}^{\infty}\left[a_{n}^{\prime}, b_{n}^{\prime}\right], 0\right)=\alpha
\end{gathered}
$$

$\bar{d}^{+}\left(\bigcup_{n=1}^{\infty}\left(\left[c_{n}, a_{n}\right] \cup\left[b_{n}, d_{n}\right]\right), 0\right)=0$ and $\bar{d}^{-}\left(\bigcup_{n=1}^{\infty}\left(\left[c_{n}^{\prime}, a_{n}^{\prime}\right] \cup\left[b_{n}^{\prime}, d_{n}^{\prime}\right]\right), 0\right)=0$. Define $f: \mathbb{R} \rightarrow \mathbb{R}$ letting

$$
f(x)=\left\{\begin{array}{l}
0 \text { for } x \in \bigcup_{n=1}^{\infty}\left(\left[a_{n}, b_{n}\right] \cup\left[a_{n}^{\prime}, b_{n}^{\prime}\right]\right) \cup\{0\}, \\
1 \text { for } x \in \mathbb{R} \backslash\left(\{0\} \cup \bigcup_{n=1}^{\infty}\left(\left[c_{n}, d_{n}\right] \cup\left[c_{n}^{\prime}, d_{n}^{\prime}\right]\right)\right), \\
\text { linear in intervals }\left[c_{n}, a_{n}\right],\left[b_{n}, d_{n}\right],\left[c_{n}^{\prime}, a_{n}^{\prime}\right],\left[b_{n}^{\prime}, d_{n}^{\prime}\right], n=1, \ldots
\end{array}\right.
$$

The function $f$ is continuous at each point except at 0 . Let $E=\bigcup_{n=1}^{\infty}\left(\left[a_{n}, b_{n}\right] \cup\right.$ $\left.\left[a_{n}^{\prime}, b_{n}^{\prime}\right]\right) \cup\{0\}$. Then $f_{\mid E}$ is constant, $\bar{d}(E, 0)=\beta$ and $\underline{d}(E, 0) \geq \alpha$. Therefore $f \in \mathcal{U C}_{\rho} \cap \mathcal{C}_{[\Lambda, \rho]}$. But, $s-\underline{d}(\{x:|f(x)|<1\}, 0)=\frac{\alpha+\beta}{2}<\rho$. Thus $f \notin s \mathcal{C}_{[\Lambda, \rho]} \cup$ $s \mathcal{U} \mathcal{C}_{\rho}$.

Example 26.38. For each $0<\Lambda \leq \rho \leq 1, \Lambda<1$ there exists $f: \mathbb{R} \rightarrow \mathbb{R}$ such that $f \in s \mathcal{C}_{[\Lambda, \rho]} \backslash \mathcal{C}_{[\Lambda, \rho]}$.

Fix $0<\Lambda<\rho \leq 1, \Lambda<1$. There exists $\alpha, \beta \in(0,1]$ such that $\frac{\alpha+\beta}{2}<\Lambda$ and $\alpha>\Lambda>\beta$. Let $\left\{\left[a_{n}, b_{n}\right]\right\}_{n \in \mathbb{N}},\left\{\left[c_{n}, d_{n}\right]\right\}_{n \in \mathbb{N}},\left\{\left[a_{n}^{\prime}, b_{n}^{\prime}\right]\right\}_{n \in \mathbb{N}},\left\{\left[c_{n}^{\prime}, d_{n}^{\prime}\right]\right\}_{n \in \mathbb{N}}$ be four sequences of closed intervals such that

$$
\ldots<c_{n}^{\prime}<a_{n}^{\prime}<b_{n}^{\prime}<d_{n}^{\prime}<c_{n+1}^{\prime}<\ldots<0<\ldots d_{n+1}<c_{n}<a_{n}<b_{n}<d_{n}<\ldots,
$$




$$
\begin{gathered}
\left.-a_{n}^{\prime}=b_{n} \text { for all } n, \bar{d}^{+}\left(\bigcup_{n=1}^{\infty}\left[a_{n}, b_{n}\right], 0\right)=\bar{d}^{-}\left(\bigcup_{n=1}^{\infty}\left[a_{n}^{\prime}, b_{n}^{\prime}\right], 0\right)\right)=1, \\
\underline{d}^{+}\left(\bigcup_{n=1}^{\infty}\left[a_{n}, b_{n}\right], 0\right)=\alpha, \underline{d}^{-}\left(\bigcup_{n=1}^{\infty}\left[a_{n}^{\prime}, b_{n}^{\prime}\right], 0\right)=\beta,
\end{gathered}
$$

$\bar{d}^{+}\left(\bigcup_{n=1}^{\infty}\left(\left[c_{n}, a_{n}\right] \cup\left[b_{n}, d_{n}\right]\right), 0\right)=0$ and $\bar{d}^{-}\left(\bigcup_{n=1}^{\infty}\left(\left[c_{n}^{\prime}, a_{n}^{\prime}\right] \cup\left[b_{n}^{\prime}, d_{n}^{\prime}\right]\right), 0\right)=0$.

Define $f: \mathbb{R} \rightarrow \mathbb{R}$ letting

$$
f(x)=\left\{\begin{array}{l}
0 \text { for } x \in \bigcup_{n=1}^{\infty}\left(\left[a_{n}, b_{n}\right] \cup\left[a_{n}^{\prime}, b_{n}^{\prime}\right]\right) \cup\{0\} \\
1 \text { for } x \in \mathbb{R} \backslash\left(\{0\} \cup \bigcup_{n=1}^{\infty}\left(\left[c_{n}, d_{n}\right] \cup\left[c_{n}^{\prime}, d_{n}^{\prime}\right]\right)\right), \\
\text { linear in intervals }\left[c_{n}, a_{n}\right],\left[b_{n}, d_{n}\right],\left[c_{n}^{\prime}, a_{n}^{\prime}\right],\left[b_{n}^{\prime}, d_{n}^{\prime}\right], n=1, \ldots
\end{array}\right.
$$

Obviously, $f$ is continuous except at 0 . Let $E=\bigcup_{n=1}^{\infty}\left(\left[a_{n}, b_{n}\right] \cup\left[a_{n}^{\prime}, b_{n}^{\prime}\right]\right) \cup\{0\}$. Then $f_{\mid E}$ is constant, $s-\bar{d}(E, 0)=1$ and $s-\underline{d}(E, 0)=\frac{\alpha+\beta}{2}>\Lambda$. Therefore $f \in \mathcal{S C}_{[\Lambda, \rho]}$. But $\underline{d}(\{x:|f(x)|<1\}, 0)=\beta<\lambda$. Thus $f \notin \mathcal{C}_{[\Lambda, \rho]}$.

\subsection{Relation between considered classes of functions for different values $\lambda$ and $\rho$}

The following proposition is obvious.

\section{Proposition 26.39.}

1. Let $0<\rho_{1} \leq \rho_{2} \leq 1$. Then $\mathcal{U C}_{\rho_{2}} \subset \mathcal{U C}_{\rho_{1}}$ and $s \mathcal{U C}_{\rho_{2}} \subset s \mathcal{U C}_{\rho_{1}}$.

2. Let $0<\Lambda_{1} \leq \rho_{1} \leq 1,0<\Lambda_{2} \leq \rho_{2} \leq 1, \Lambda_{1} \leq \Lambda_{2}$ and $\rho_{1} \leq \rho_{2}$. Then $\mathcal{C}_{\left[\Lambda_{2}, \rho_{2}\right]} \subset$ $\mathcal{C}_{\left[\Lambda_{1}, \rho_{1}\right]}$ and $s \mathcal{C}_{\left[\Lambda_{2}, \rho_{2}\right]} \subset s \mathcal{C}_{\left[\Lambda_{1}, \rho_{1}\right]}$.

Example 26.40. For each $0<\rho_{1}<\rho_{2} \leq 1$ there exists $f \in\left(\mathcal{U C}_{\rho_{1}} \cap s \mathcal{U C} \mathcal{C}_{1}\right) \backslash$ $\left(\mathcal{U C}_{\rho_{2}} \cup s \mathcal{U C}_{\rho_{2}}\right)$. Moreover, for each $0<\Lambda_{2} \leq \rho_{2}$, we have $f \in\left(\mathcal{C}_{\left[\rho_{1}, \rho_{1}\right]} \cap\right.$ $\left.s \mathcal{C}_{\left[\rho_{1}, \rho_{1}\right]}\right) \backslash\left(\mathcal{C}_{\left[\Lambda_{2}, \rho_{2}\right]} \cup s \mathcal{C}_{\left[\Lambda_{2}, \rho_{2}\right]}\right)$.

Let $\left\{\left[a_{n}, b_{n}\right]\right\}_{n \in \mathbb{N}}$ and $\left\{\left[c_{n}, d_{n}\right]\right\}_{n \in \mathbb{N}}$ be two sequences of closed intervals such that $0<\ldots d_{n+1}<c_{n}<a_{n}<b_{n}<d_{n}<\ldots<d_{1}, d^{+}\left(\bigcup_{n=1}^{\infty}\left[a_{n}, b_{n}\right], 0\right)=$ $\frac{\rho_{1}+\rho_{2}}{2}$ and $\bar{d}^{+}\left(\bigcup_{n=1}^{\infty}\left(\left[c_{n}, a_{n}\right] \cup\left[b_{n}, d_{n}\right]\right), 0\right)=0$. Define $f: \mathbb{R} \rightarrow \mathbb{R}$ letting 


$$
f(x)=\left\{\begin{array}{l}
0 \text { for } x \in \bigcup_{n=1}^{\infty}\left(\left[a_{n}, b_{n}\right] \cup\left[-b_{n},-a_{n}\right]\right) \cup\{0\}, \\
1 \text { for } x \in \mathbb{R} \backslash\left(\{0\} \cup \bigcup_{n=1}^{\infty}\left(\left[c_{n}, d_{n}\right] \cup\left[-d_{n},-c_{n}\right]\right)\right), \\
\text { linear in intervals }\left[c_{n}, a_{n}\right],\left[b_{n}, d_{n}\right],\left[-a_{n},-c_{n}\right],\left[-d_{n},-b_{n}\right], n=1, \ldots
\end{array}\right.
$$

It is clear that $f$ is continuous at each point except at 0 . Let $E=\{0\} \cup$ $\bigcup_{n=1}^{\infty}\left[a_{n}, b_{n}\right]$. Then $f_{\mid E}$ is constant and $d(E, 0)=s-d(E, 0)=\frac{\rho_{1}+\rho_{2}}{2}>\rho_{1}$. Therefore $f \in s \mathcal{U C}_{\rho} \cap \mathcal{U} \mathcal{C}_{\rho} \cap \mathcal{C}_{\left[\rho_{1}, \rho_{1}\right]} \cap s \mathcal{C}_{\left[\rho_{1}, \rho_{1}\right]}$. On the other hand,

$$
\begin{aligned}
\underline{d}(\{x:|f(x)|<1\}, 0) & =s-\underline{d}(\{x:|f(x)|<1\}, 0) \leq \bar{d}\left(\bigcup_{n=1}^{\infty}\left(\left[c_{n}, d_{n}\right] \cup\left[-d_{n},-b_{n}\right]\right)=\right. \\
& =\frac{\rho_{1}+\rho_{2}}{2}<\rho_{2} .
\end{aligned}
$$

Thus $f \notin \mathcal{U} \mathcal{C}_{\rho_{2}} \cup s \mathcal{U} \mathcal{C}_{\rho_{2}} \cup \mathcal{C}_{\left[\Lambda_{2}, \rho_{2}\right]} \cup s \mathcal{C}_{\left[\Lambda_{2}, \rho_{2}\right]}$ for any $\Lambda_{2} \in\left(0, \rho_{2}\right]$.

From Proposition 26.39 and Example 26.40 we have.

\section{Theorem 26.41.}

1. Let $\rho_{1}, \rho_{2} \in(0,1]$. Then $\mathcal{U} \mathcal{C}_{\rho_{2}} \subset \mathcal{U} \mathcal{C}_{\rho_{1}}$ if and only if $\rho_{1} \leq \rho_{2}$. Moreover, if $\rho_{1}<\rho_{2}$ then $\mathcal{U C} \mathcal{\rho}_{2} \varsubsetneqq \mathcal{U C} \mathcal{C}_{\rho_{1}}$.

2. Let $\rho_{1}, \rho_{2} \in(0,1]$. Then $s \mathcal{U} \mathcal{C}_{\rho_{2}} \subset s \mathcal{U} \mathcal{C}_{\rho_{1}}$ if and only if $\rho_{1} \leq \rho_{2}$. Moreover, if $\rho_{1}<\rho_{2}$ then $s \mathcal{U} \mathcal{C}_{\rho_{2}} \varsubsetneqq s \mathcal{U C} \rho_{\rho_{1}}$.

Example 26.42. Let $0<\Lambda_{1}<\rho_{1}<1$. For each $\Lambda_{1}<\Lambda_{2} \leq 1$ there exists $f \in$ $\left(\mathcal{C}_{\left[\Lambda_{1}, \rho_{1}\right]} \cap s \mathcal{C}_{\left[\Lambda_{1}, \rho_{1}\right]}\right) \backslash\left(\mathcal{C}_{\left[\Lambda_{2}, \Lambda_{2}\right]} \cup s \mathcal{C}_{\left[\Lambda_{2}, \Lambda_{2}\right]}\right)$.

Let $\left\{\left[a_{n}, b_{n}\right]\right\}_{n \in \mathbb{N}}$ and $\left\{\left[c_{n}, d_{n}\right]\right\}_{n \in \mathbb{N}}$ be two sequences of closed intervals such that $0<\ldots d_{n+1}<c_{n}<a_{n}<b_{n}<d_{n}<\ldots<d_{1}, \underline{d}^{+}\left(\bigcup_{n=1}^{\infty}\left[a_{n}, b_{n}\right], 0\right)=$ $\frac{\Lambda_{1}+\Lambda_{2}}{2}, \bar{d}^{+}\left(\bigcup_{n=1}^{\infty}\left[a_{n}, b_{n}\right], 0\right)=1$ and $\bar{d}^{+}\left(\bigcup_{n=1}^{\infty}\left(\left[c_{n}, a_{n}\right] \cup\left[b_{n}, d_{n}\right]\right), 0\right)=0$. Define $f: \mathbb{R} \rightarrow \mathbb{R}$ letting

$$
f(x)=\left\{\begin{array}{l}
0 \text { for } x \in \bigcup_{n=1}^{\infty}\left(\left[a_{n}, b_{n}\right] \cup\left[-b_{n},-a_{n}\right]\right) \cup\{0\}, \\
1 \text { for } x \in \mathbb{R} \backslash\left(\{0\} \cup \bigcup_{n=1}^{\infty}\left(\left[c_{n}, d_{n}\right] \cup\left[-d_{n},-c_{n}\right]\right)\right), \\
\text { linear in intervals }\left[c_{n}, a_{n}\right],\left[b_{n}, d_{n}\right],\left[-a_{n},-c_{n}\right],\left[-d_{n},-b_{n}\right], n=1, \ldots
\end{array}\right.
$$

It is clear that $f$ is continuous at each point except at 0 . Let $E=\{0\} \cup$ $\bigcup_{n=1}^{\infty}\left(\left[a_{n}, b_{n}\right] \cup\left[-b_{n},-a_{n}\right]\right)$. Then $f_{\mid E}$ is constant $\underline{d}(E, 0)=s-\underline{d}(E, 0)=\frac{\Lambda_{1}+\Lambda_{2}}{2}>$ 
$\Lambda_{1}$ and $\bar{d}(E, 0)=s-\bar{d}(E, 0)=1$. Hence $f \in \mathcal{C}_{\left[\Lambda_{1}, \rho_{1}\right]} \cap s \mathcal{C}_{\left[\Lambda_{1}, \rho_{1}\right]}$. On the other hand,

$$
\begin{aligned}
\underline{d}(\{x:|f(x)|<1\}, 0) & =s-\underline{d}(\{x:|f(x)|<1\}, 0) \leq \\
& \left.\leq \underline{\bigcup}_{n=1}^{\infty}\left(\left[c_{n}, d_{n}\right] \cup\left[-d_{n},-c_{n}\right]\right), 0\right)=\frac{\Lambda_{1}+\Lambda_{2}}{2}<\Lambda_{2} .
\end{aligned}
$$

Thus $f \notin \mathcal{C}_{\left[\Lambda_{2}, \Lambda_{2}\right]} \cup s \mathcal{C}_{\left[\Lambda_{2}, \Lambda_{2}\right]}$.

From Proposition 26.39 and Examples 26.42 and 26.40 we have.

\section{Theorem 26.43.}

1. Let $0<\Lambda_{1} \leq \rho_{1} \leq 1$ and $0<\Lambda_{2} \leq \rho_{2} \leq 1$. Then $\mathcal{C}_{\left[\Lambda_{2}, \rho_{2}\right]} \subset \mathcal{C}_{\left[\Lambda_{1}, \rho_{1}\right]}$ if and only if $\Lambda_{1} \leq \Lambda_{2}$ and $\rho_{1} \leq \rho_{2}$. Moreover, if $\Lambda_{1}<\Lambda_{2}$ or $\rho_{1}<\rho_{2}$ then $\mathcal{C}_{\left[\Lambda_{2}, \rho_{2}\right]} \varsubsetneqq$ $\mathcal{C}_{\left[\Lambda_{1}, \rho_{1}\right]}$.

2. Let $0<\Lambda_{1} \leq \rho_{1} \leq 1$ and $\left.0<\Lambda_{2} \leq \rho_{2} \leq 1\right]$. Then $s \mathcal{C}_{\left[\Lambda_{2}, \rho_{2}\right]} \subset s \mathcal{C}_{\left[\Lambda_{1}, \rho_{1}\right]}$ if and only if $\Lambda_{1} \leq \Lambda_{2}$ and $\rho_{1} \leq \rho_{2}$. Moreover, if $\Lambda_{1}<\Lambda_{2}$ or $\rho_{1}<\rho_{2}$ then $s \mathcal{C}_{\left[\Lambda_{2}, \rho_{2}\right]} \varsubsetneqq s \mathcal{C}_{\left[\Lambda_{1}, \rho_{1}\right]}$.

Example 26.44. Let $\left.0<\Lambda_{1} \leq \rho_{1} \leq 1\right]$ and $0<\rho_{2} \leq 1$. Then there exists $f \in\left(\mathcal{U C}_{\rho_{2}} \cap s \mathcal{U} \mathcal{C}_{\rho_{2}}\right) \backslash\left(\mathcal{C}_{\left[\Lambda_{1}, \rho_{1}\right]} \cup s \mathcal{C}_{\left[\Lambda_{1}, \rho_{1}\right]}\right)$

Let $\left\{\left[a_{n}, b_{n}\right]\right\}_{n \in \mathbb{N}}$ and $\left\{\left[c_{n}, d_{n}\right]\right\}_{n \in \mathbb{N}}$ be two sequences of closed intervals such that $0<\ldots d_{n+1}<c_{n}<a_{n}<b_{n}<d_{n}<\ldots<d_{1}, \underline{d}^{+}\left(\bigcup_{n=1}^{\infty}\left[a_{n}, b_{n}\right], 0\right)=0$, $\bar{d}^{+}\left(\bigcup_{n=1}^{\infty}\left[a_{n}, b_{n}\right], 0\right)=1$ and $\bar{d}^{+}\left(\bigcup_{n=1}^{\infty}\left(\left[c_{n}, a_{n}\right] \cup\left[b_{n}, d_{n}\right]\right), 0\right)=0$. Define $f: \mathbb{R} \rightarrow \mathbb{R}$ letting

$$
f(x)=\left\{\begin{array}{l}
0 \text { for } x \in \bigcup_{n=1}^{\infty}\left(\left[a_{n}, b_{n}\right] \cup\left[-b_{n},-a_{n}\right]\right) \cup\{0\}, \\
1 \text { for } x \in \mathbb{R} \backslash\left(\{0\} \cup \bigcup_{n=1}^{\infty}\left(\left[c_{n}, d_{n}\right] \cup\left[-d_{n},-c_{n}\right]\right)\right), \\
\text { linear in intervals }\left[c_{n}, a_{n}\right],\left[b_{n}, d_{n}\right],\left[-a_{n},-c_{n}\right],\left[-d_{n},-b_{n}\right], n=1, \ldots
\end{array}\right.
$$

Clearly, $f$ is continuous at each point except at 0 . Let $E=\{0\} \cup \bigcup_{n=1}^{\infty}\left(\left[a_{n}, b_{n}\right] \cup\right.$ $\left.\left[-b_{n},-a_{n}\right]\right)$. Then $f_{\mid E}$ is constant $\underline{d}(E, 0)=s-\underline{d}(E, 0)=0$ and $\bar{d}(E, 0)=$ $s-\bar{d}(E, 0)=1$. Hence $f \in \mathcal{U} \mathcal{C}_{\rho_{2}} \cap \mathcal{S U C}_{\rho_{2}}$. On the other hand, 


$$
\begin{aligned}
\underline{d}(\{x:|f(x)|<1\}, 0) & =s \underline{d}(\{x:|f(x)|<1\}, 0) \leq \\
& \leq \underline{d}\left(\bigcup_{n=1}^{\infty}\left(\left[c_{n}, d_{n}\right] \cup\left[-d_{n},-c_{n}\right]\right), 0\right)=0 .
\end{aligned}
$$

Thus $f \notin \mathcal{C}_{\left[\Lambda_{1}, \rho_{1}\right]} \cup s \mathcal{C}_{\left[\Lambda_{1}, \rho_{1}\right]}$.

Example 26.45. Let $0<\rho_{2}<\rho_{1} \leq 1$. Then for each $0<\Lambda \leq \rho_{2}$ there exists $f \in\left(\mathcal{C}_{\left[\Lambda, \rho_{2}\right]} \cap s \mathcal{C}_{\left[\Lambda, \rho_{2}\right]}\right) \backslash\left(\mathcal{U C} \mathcal{C}_{\rho_{1}} \cup s \mathcal{U C} \mathcal{C}_{\rho_{1}}\right)$

Let $\left\{\left[a_{n}, b_{n}\right]\right\}_{n \in \mathbb{N}}$ and $\left\{\left[c_{n}, d_{n}\right]\right\}_{n \in \mathbb{N}}$ be two sequences of closed intervals such that $0<\ldots d_{n+1}<c_{n}<a_{n}<b_{n}<d_{n}<\ldots<d_{1}, d^{+}\left(\bigcup_{n=1}^{\infty}\left[a_{n}, b_{n}\right], 0\right)=$ $\frac{\rho_{1}+\rho_{2}}{2}$ and $\bar{d}^{+}\left(\bigcup_{n=1}^{\infty}\left(\left[c_{n}, a_{n}\right] \cup\left[b_{n}, d_{n}\right]\right), 0\right)=1$. Define $f: \mathbb{R} \rightarrow \mathbb{R}$ letting

$$
f(x)=\left\{\begin{array}{l}
0 \text { for } x \in \bigcup_{n=1}^{\infty}\left(\left[a_{n}, b_{n}\right] \cup\left[-b_{n},-a_{n}\right]\right) \cup\{0\}, \\
1 \text { for } x \in \mathbb{R} \backslash\left(\{0\} \cup \bigcup_{n=1}^{\infty}\left(\left[c_{n}, d_{n}\right] \cup\left[-d_{n},-c_{n}\right]\right)\right), \\
\text { linear in intervals }\left[c_{n}, a_{n}\right],\left[b_{n}, d_{n}\right],\left[-a_{n},-c_{n}\right],\left[-d_{n},-b_{n}\right], n=1, \ldots
\end{array}\right.
$$

The function $f$ is continuous at each point except at 0 . Let $E=\{0\} \cup$ $\bigcup_{n=1}^{\infty}\left(\left[a_{n}, b_{n}\right] \cup\left[-b_{n},-a_{n}\right]\right)$. Then $f_{\mid E}$ is constant $d(E, 0)=s-d(E, 0)=\frac{\rho_{1}+\rho_{2}}{2}>$ $\rho_{2}$. Hence $f \in \mathcal{C}_{\left[\Lambda, \rho_{2}\right]} \cap s \mathcal{C}_{\left[\Lambda, \rho_{2}\right]}$. On the other hand,

$$
\begin{aligned}
d(\{x:|f(x)|<1\}, 0) & =s-d(\{x:|f(x)|<1\}, 0)= \\
& =\underline{d}\left(\bigcup_{n=1}^{\infty}\left(\left[c_{n}, d_{n}\right] \cup\left[-d_{n},-c_{n}\right]\right), 0\right)=\frac{\rho_{1}+\rho_{2}}{2}<\rho_{1} .
\end{aligned}
$$

Thus $f \notin \mathcal{U} \mathcal{C}_{\rho_{1}} \cup s \mathcal{U} \mathcal{C}_{\rho_{1}}$.

From Proposition 26.39 and Examples 26.44 and 26.45 we have.

\section{Theorem 26.46.}

1. Let $0<\Lambda_{1} \leq \rho_{1} \leq 1$ and $0<\rho_{2} \leq 1$. Then $\mathcal{U} \mathcal{C}_{\rho_{2}} \subset \mathcal{C}_{\left[\Lambda_{1}, \rho_{1}\right]}$ if and only if

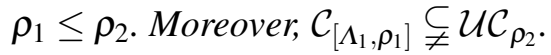

2. Let $0<\Lambda_{1} \leq \rho_{1} \leq 1$ and $0<\rho_{2} \leq 1$. Then $s \mathcal{U C}_{\rho_{2}} \subset s \mathcal{C}_{\left[\Lambda_{1}, \rho_{1}\right]}$ if and only if $\rho_{1} \leq \rho_{2}$. Moreover, $s \mathcal{C}_{\left[\Lambda_{1}, \rho_{1}\right]} \varsubsetneqq s \mathcal{U} \mathcal{C}_{\rho_{2}}$. 


\title{
References
}

[1] A. Alikhani, Borel measurability of extreme path derivatieves, Real Anal. Exchange 12 (1986-87), 216-246.

[2] K. Banaszewski, Funkcje ciagłe wzgledem systemu ścieżek, Doctoral Thesis, Łódź, 1995.

[3] A. M. Bruckner, Differentiation of Real Functions, Lecture Notes in Mathematics, Vol. 659, Springer-Verlag Berlin Heidelberg New York, 1978.

[4] A. M. Bruckner, R. J. O'Malley, B. S. Thomson, Path Derivatives: A Unified View of Certain Generalized Derivatives, Trans. Amer. Math. Soc. 283 (1984), 97-125.

[5] J. Jȩdrzejewski, On limit numbers of real functions, Fund. Math. 83(3) (1973/74), 269-281.

[6] A. Karasiǹska, E. Wagner-Bojakowska, Some remarks on $\rho$-upper density, Tatra Mt. Math. Publ. 46 (2010), 85-89.

[7] S. Kowalczyk, K. Nowakowska, A note on $\rho$-upper continuous functions, Tatra Mt. Math. Publ. 44 (2009), 153-158.

[8] S. Kowalczyk, K. Nowakowska, Maximal classes for the family of $[\lambda, \rho]$-continuous functions, Real Anal. Exchange 36 (2010-11), 307-324.

[9] S. Kowalczyk, K. Nowakowska, Maximal classes for $\rho$-upper continuous functions, Journal of Applied Analysis 19 (2013), 69-89.

[10] M. Marciniak, R. Pawlak, On the restrictions of functions. Finitely continuous functions and path continuity, Tatra Mt. Math. Publ. 24 (2002), 65-77.

[11] M. Marciniak, On path continuity, Real Anal. Exchange 29(1) (2003-2004), 247-255.

[12] J. Masterson, A nonstandard result about path continuity, Acta Math. Hungar. 59, Issue 1-2, 1992, 147-149.

[13] K. Nowakowska, On the family of $[\Lambda, \rho]$-continuous functions, Tatra Mt. Math. Publ. 44 (2009), 129-138.

[14] F. D. Tall, The density topology, Pacific Journal of Mathematics 62(1) (1976), 275-284.

[15] B. S. Thomson, Real Functions, Lecture Notes in Mathematics, Vol. 1170, SpringerVerlag Berlin Heidelberg New York, 1985.

[16] W. Wilczyǹski, Density topologies, Handbook of Measure Theory, chapter 15, Elsevier 2012, 307-324.

\section{STANISEW KOWALCZYK \\ Institute of Mathematics, Pomeranian Academy ul. Arciszewskiego 22d, 76-200 Słupsk, Poland \\ E-mail: stkowalczeonet.eu}

\author{
KATARZYNA NOWAKOWSKA \\ Institute of Mathematics, Pomeranian Academy \\ ul. Arciszewskiego 22d, 76-200 Słupsk, Poland \\ E-mail: nowakowska_k@go2.pl
}

